\title{
ON COMPLETENESS OF THE PRODUCTS OF HARMONIC FUNCTIONS
}

\author{
A. G. RAMM
}

AbSTRACT. Let $L$ be a partial differential operator in $R^{n}$ with constant coefficients. We prove that, under some assumption on $L$, the set of products of the elements of the null-space of $L$ forms a complete set in $L^{p}(D), p \geqslant 1$, where $D$ is any bounded domain. In particular, the products of harmonic functions form a complete set in $L^{p}(D), p \geqslant 1$.

Let $D$ be a bounded domain, $u_{l m}=r^{l} Y_{l m}(\omega), l \geqslant 0$, where $Y_{l m}(\omega)$ are the spherical harmonics, $f \in L^{2}(D)$, and $\int_{D} f(x) u_{l_{1} m_{1}}(x) u_{l_{2} m_{2}}(x) d x=0, \forall l_{1}, l_{2},\left|m_{1}\right|$ $\leqslant l_{1},\left|m_{2}\right| \leqslant l_{2}$. Then $f=0$.

1. Introduction. Let $D \subset R^{3}$ be a bounded domain with the origin inside $D$. The functions $u_{l m}=r^{l} Y_{l m}(\omega)$, where $Y_{l m}$ are the normalized spherical harmonics, $l=$ $0,1,2, \ldots,-l \leqslant m \leqslant l, \int_{S^{2}} Y_{l m}(\omega) \bar{Y}_{l^{\prime} m^{\prime}}(\omega) d \omega=\delta_{l l^{\prime}} \delta_{m m^{\prime}}$, and $S^{2}$ is the unit sphere. The functions $u_{l m}$ form a complete set of harmonic functions. The question we are concerned with is completeness of the system $u_{l_{1} m_{1}} u_{l_{2} m_{2}}$ in $L^{2}(D)$. The result is

THEOREM 1. Let

(1) $\int_{D} f(x) u_{l_{1} m_{1}}(x) u_{l_{2} m_{2}}(x) d x=0, \quad l_{1}, l_{2}=0,1,2, \ldots,\left|m_{1}\right| \leqslant l_{1},\left|m_{2}\right| \leqslant l_{2}$.

Then $f=0$.

In $\$ 2$ we prove Theorem 1 and in $\$ 3$ a more general Theorem 2.

2. Proof. Let $B_{R}=\{x:|x| \leqslant R\}, B_{R} \supset D$. Extend $f$ to $B_{R}$ by setting $f=0$ in $B_{R} \backslash D$. Write (1) as

$$
\int_{0}^{R} d r r^{2+l_{1}+l_{2}} \int_{S^{2}} f(r, \omega) Y_{l_{1} m_{1}}(\omega) Y_{l_{2} m_{2}}(\omega) d \omega=0
$$

One has [1]

$$
Y_{l_{1} m_{1}}(\omega) Y_{l_{2} m_{2}}(\omega)=\sum_{l=\left|l_{1}-l_{2}\right|}^{l_{1}+l_{2}} \rho\left(l_{1}, l_{2}, l\right)\left(l_{1} l_{2} m_{1} m_{2} \mid l m\right) Y_{l m}(\omega),
$$

Received by the editors September 5, 1985.

1980 Mathematics Subject Classification (1985 Revision). Primary 35C10.

C1986 American Mathematical Society $0002-9939 / 86 \$ 1.00+\$ .25$ per page 
where $\left(l_{1} l_{2} m_{1} m_{2} \mid l m\right)$ are the Clebsch-Gordon coefficients, and $\rho\left(l_{1}, l_{2}, l\right)=$ $\left\{\left(2 l_{1}+1\right)\left(2 l_{2}+1\right) /(2 l+1)\right\}^{1 / 2}\left(l_{1} l_{2} 00 \mid l 0\right)$. It follows from (3) that

$$
\int_{S^{2}} Y_{l_{1} m_{1}}(\omega) Y_{l_{2} m_{2}}(\omega) \bar{Y}_{l_{3} m_{3}}(\omega) d \omega=\rho\left(l_{1}, l_{2}, l_{3}\right)\left(l_{1} l_{2} m_{1} m_{2} \mid l_{3} m_{3}\right) \text {. }
$$

It follows from (2) and (4) that

$$
\int_{0}^{R} d r r^{2+l_{1}+l_{2}} \sum_{l=\left|l_{1}-l_{2}\right|}^{l_{1}+l_{2}} f_{l m}(r) \rho\left(l_{1}, l_{2}, l\right)\left(l_{1} l_{2} m_{1} m_{2} \mid l m\right)=0 .
$$

Multiply (5) by $\left(l_{1} l_{2} m_{1} m_{2} \mid L M\right)$, sum over $m_{1}$ and $m_{2}$, and use the formula [1]:

$$
\sum_{\substack{m_{1}, m_{2} \\ m_{1}+m_{2}=m}}\left(l_{1} l_{2} m_{1} m_{2} \mid l m\right)\left(l_{1} l_{2} m_{1} m_{2} \mid l^{\prime} m^{\prime}\right)=\delta_{l l^{\prime}} \delta_{m m^{\prime}}
$$

to obtain

$$
\int_{0}^{R} d r r^{2+l_{1}+l_{2}} f_{L M}(r) \rho\left(l_{1}, l_{2}, L\right)=0 .
$$

where $f_{L M}=\int_{S^{2}} f(r, \omega) \bar{Y}_{L M}(\omega) d \omega$. The coefficient $\rho\left(l_{1}, l_{2}, L\right)=0$ unless $l_{1}+l_{2}+$ $L$ is even and $\left|l_{1}-l_{2}\right| \leqslant L \leqslant l_{1}+l_{2}$. Take $l_{2}=l_{1}+L$. Then $\rho\left(l_{1}, l_{1}+L, L\right) \neq 0$. Thus (7) yields

$$
\int_{0}^{R} d r r^{2+2 l_{1}+L} f_{L M}(r)=0, \quad l_{1}=0,1,2, \ldots
$$

From (8) and Müntz's theorem [2] it follows that $f_{L M}(r)=0$. Thus $f=0$.

It is probably true that if $\mathscr{L} u_{n}=0$, where $\mathscr{L}$ is an elliptic operator of the second order in $B_{R}$ and the system $\left\{u_{n}\right\}$ is a complete system of the solutions to the equation $L u=0$ in $B_{R}$, then the system $u_{n}(x) u_{n^{\prime}}(x)$ is complete in $L^{2}(D)$, $D \subset B_{R}$. The system $\left\{u_{n}\right\}$ is a complete system of the solutions to the equation $L u=0$ in $B_{R}$ if the closure of the linear span of $u_{n}$ is the null space of $L$.

3. Generalizations. In this section we outline another approach to the problem. This approach is more general and leads to the following result. Let

$$
L u=\sum_{|\alpha| \leqslant l} a_{\alpha} D^{\alpha} u(x), \quad x \in R^{n}, n \geqslant 2,
$$

where $a_{\alpha}=$ const, $\alpha$ is a multi-index, $l>1$. Let

$$
M=\left\{z: z \in C^{n}, \sum_{|\alpha| \leqslant l} a_{\alpha} z^{\alpha}=0\right\} .
$$

Assumption A. There exist two points $a$ and $b, a, b \in M$, such that the tangent planes $T_{a}$ and $T_{b}$ to $M$ at the points $a$ and $b$ are not parallel.

Let $N(L)=\{u: L u=0\}, B_{R}=\left\{x:|x| \leqslant R, x \in R^{n}\right\}$, and $B_{\varepsilon}(a)=\{z:|z-a|$ $\left.\leqslant \varepsilon, z \in C^{n}\right\}$.

Theorem 2. Suppose that Assumption A holds, $f \in L^{p}\left(B_{R}\right), p \geqslant 1, R>0$ is an arbitrary fixed number, and

$$
\int f u_{j} u_{j^{\prime}} d x=0, \quad \forall u_{j}, u_{j^{\prime}} \in N(L), \int=\int_{B_{R}}
$$

Then $f=0$. 
REMARK 1. The conclusion remains valid if $u_{j}, u_{j^{\prime}}$ run through a linear subset of $N(L)$ which is dense in $N(L)$ in $L^{p}$ norm.

EXAMPLE. If $L u=\Delta u, x \in R^{3}$, then

$$
M=\left\{z: \sum_{j=1}^{3} z_{j}^{2}=0\right\} .
$$

Clearly Assumption A holds, e.g., for $a=(1,0, i), b=(0,1, i)$. Linear combinations of the functions $r^{m} Y_{m}, m \geqslant 0$, are dense in $N(\Delta)$ in $L^{p}\left(B_{R}\right)$. Therefore, Theorem 2 and Remark 1 imply the conclusion of Theorem 1.

REMARK 2. Note that the operator $L$ in Theorem 2 can be elliptic, hyperbolic, parabolic, or neither. The proof of Theorem 2 is based on the following lemma:

LEMMA 1. Let $M$ be a differentiable manifold in $C^{n}$ of dimension $\tau=n-1$, and Assumption A holds. Then, for any $\varepsilon>0$, the set $\{x+y\}, x \in M \cap B_{\varepsilon}(a), y \in M$ $\cap B_{\varepsilon}(b)$, contains a ball $B_{\delta}(a+b) \subset C^{n}$, where $\delta=\delta(\varepsilon)>0$.

Proof of Lemma 1. Consider the mapping $f: C^{n} \times C^{n} \rightarrow C^{n}$ given by the formula $f(x, y)=x+y$. This mapping is linear in $x$ and $y$. Therefore its differential coincides with the mapping. The restriction of $d f$ on $M \times M$ is defined, in particular, on $T_{a} \times T_{b}$. If $T_{a}$ is not parallel to $T_{b}$ then the set $\{x+y\}$ contains a ball $B_{\delta}(a+b)$ if $x$ runs through $T_{a} \cap B_{\varepsilon}(a)$ and $y$ runs through $T_{b} \cap B_{\varepsilon}(b)$. For sufficiently small $\varepsilon>0$ the elements of $T_{a} \cap B_{\varepsilon}(a)$ differ very little from the elements of $M \cap B_{\varepsilon}(a)$. Therefore the conclusion of Lemma 1 holds.

REMARK 3. If $\operatorname{dim} M_{1}=\tau_{1}, \operatorname{dim} M_{2}=\tau_{2}$, and $\operatorname{rank}\left(T_{a_{1}}, T_{a_{2}}\right)=n$, then the set $\{x+y\}$, where $x \in M_{1} \cap B_{\varepsilon}\left(a_{1}\right)$ and $y \in M_{2} \cap B_{\varepsilon}\left(a_{2}\right)$, contains a ball $B_{\delta}\left(a_{1}+a_{2}\right), \delta=\delta(\varepsilon)>0$. Here $\left(T_{a_{1}}, T_{a_{2}}\right)$ is the union of the systems of the basis vectors in $T_{a_{1}}$ and $T_{a_{2}}$.

ProOF OF THEOREM 2. If

$$
z \in M=\left\{z: \sum_{|\alpha| \leqslant l} a_{\alpha} z^{\alpha}=0\right\},
$$

then $\exp (x \cdot z) \in N(L)$, where

$$
x \cdot z=\sum_{j=1}^{n} x_{j} z_{j}
$$

Consider $\exp \{x(z+v)\}$, where $z, v \in M$. By Lemma 1, the set $\{z+v\} \supset$ $B_{\delta}(a+b)$, where $a$ and $b$ belong to $M$ and $T_{a}$ is not parallel to $T(b)$. Assume that

$$
\int f(x) \exp \{x(z+v)\} d x=0, \quad \forall z, v \in M .
$$

Then

$$
F(p) \triangleq \int f(x) \exp (p \cdot x) d x=0, \quad \forall p \in B_{\delta}(a+b) .
$$

Since $F(p)$ is an entire function of $p$, we conclude that $F(p) \equiv 0$ and $f(x)=0$. 
REMARK 4. Our argument shows that the assumption $f \in L^{p}\left(B_{R}\right), p \geqslant 1$, can be relaxed: $f \in S^{\prime}, \operatorname{supp} f \subset B_{R}$ is sufficient, where $S$ is the set of $C_{0}^{\infty}\left(R^{n}\right)$ test functions, and $S^{\prime}$ is the corresponding space of distributions.

REMARK 5. If

$$
\sum_{|\alpha| \leqslant l} a_{\alpha} z^{\alpha}=\left(\sum_{j=1}^{n} c_{j} z_{j}\right)^{l},
$$

then Assumption A does not hold.

REMARK 6. It is interesting to prove Theorem 2 in the case when $a_{\alpha}=a_{\alpha}(x)$. This is an open problem.

REMARK 7. The results of this paper are useful in the study of multidimensional inverse problems [3, Chapter 6].

The idea that the set $\{z+v\}$ in the proof of Theorem 2 should contain an open set was suggested by Professor H. S. Shapiro with whom the author discussed the problem. The author is grateful to Professor H. S. Shapiro for the valuable suggestion and discussion, and to ONR for support.

\section{REFERENCES}

1. G. Lubarskii, Group theory and its application in physics, Fizmatgiz, Moscow, 1957.

2. N. Akhiezer, Theory of approximation, Ungar, New York, 1956.

3. A. G. Ramm, Scattering by obstacles, Reidel, Dordrecht, 1986.

Department of Mathematics, KanSas State University, Manhattan, KanSas 66506 